\title{
Subacute thyroiditis due to seasonal influenzae vaccination
}

\author{
Fatma Aybala Altay ${ }^{1}$ \\ Galip Güz² \\ Mustafa Altay ${ }^{3}$ \\ ${ }^{1}$ Ankara Dışkapı Yıldırım Beyazıd Education and Research Hospital, Department of Infectious Disease and Microbiology \\ ${ }^{2}$ Gazi University Medical Faculty, Department of Nephrology, Ankara, Turkey \\ ${ }^{3}$ Keçiören Education and Research Hospital, Department of Endocrinology and Metabolism, Ankara, Turkey
}

Introduction: Subacute thyroiditis due to vaccination is reported in only a few case reports. A peritoneal dialysis patient who experienced a repeating attack after a vaccination for influenzae while she was being followed and treated succesfully for subacute thyroiditis is presented.

Case: Twenty eight years old female patient who has been applying dialysis for nine years was seen in policlinic telling about upper respiratory tract infection 2 months ago and pain at her thyroid lodge for 3 weeks. Her thyroid was palpable and tender. She was diagnosed as subacute thyroiditis with high levels of ESR, CRP, TSH, anti TG, TPO and consistent ultrasonography findings. She was begun ibubrofen at $1800 \mathrm{mg} / \mathrm{day}$ in three divided doses after consulting with nephrology. She had recovered totally a week after and begun to follow up with levotyroxine $75 \mathrm{mcg} / \mathrm{day}$. She applied to hospial three months later with fatigue, fever, sore throat, dispnea and pain and swolling at her thyroid lodge again.

USG showed a larger thyroid. ESR,CRP, anti TG and anti TPO were much more higher. It was learnt that she had been vaccinated for influenzae 3 weeks ago and began to suffer from gribal symptoms at the third day and pain and swelling of thyroid at the second week of vaccination. Her thyroid scintigraphy showed heterogenous and lower activity and her thyroid biopsy resulted as consistent with subacute thyroiditis. So the patient was diagnosed as subacute thyroiditis and ibubrofen was begun besides raising levothyroxine dose to $100 \mathrm{mcg} / \mathrm{day}$. Methyl prednisolone of $40 \mathrm{mg} /$ day was added because there was no symptomatic improvement at the third day. The symtoms regressed after steroid in three days and recovered totally at the end of the first week. Steroid was stopped by gradually declining at the third week.

Discussion: Influenza like symptoms are known to develop after vaccination. This case is showing a rare condition due to vaccination to cause a subacute thyroiditis just like the virus can make. 